Die Kosten betragen wohl etwas mehr, als diejenigen für gewöhnliche Reservoirs, aber die Vortheile sind grösser. Man hat bei dieser Einrichtung keine Keller, keine Pumpen, überhaupt keine besondere Vorsicht nöthig, wodurch die Assecuranz viel niedriger zu stehen kommt.

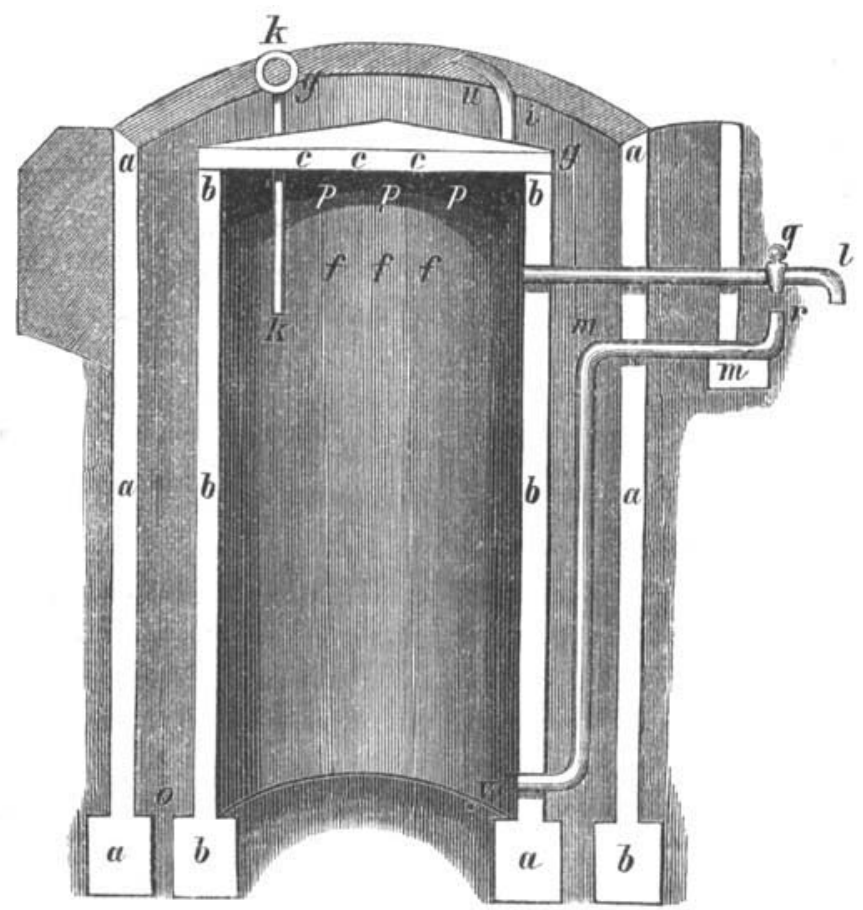

\title{
Ueber die Behandlung des Fleisches in Urugnay
}

zur Versendung anf den europäischen Markt, so wie über die verschiedenen Bereitungsmethoden des Fleischextracts daselbst.

\section{Von Demselben.}

Bei den Verwüstungen, welche die Viehsenche in Europa hervorgebracht und den in Folge derselben entstandenen erhöheten Fleischpreisen ist es von der grössten Wichtigkeit, 
das Augenmerk auf den grossen Ueberfluss an Fleisch in den reichen Weiden des La Platastromes zu lenken. Es ist noch nicht lange her, dass dort das Vieh blos seiner Häute wegen geschätzt wurde, während das Fleisch entweder verloren ging oder zu sehr geringen Preisen verkauft wurde. Selbst jetzt noch wird auf dern Markte zu Buenos Ayres das Fleisch erster Qualität stiickweise verkauft, aber nicht nach dem Gewichte. Eine Schöpsenkenle kostet daselbst etwa 6-7 Silbergroschen. Rindfleisch steht in gleichem Preise, meist niedriger.

Die Zahl des Hornvieh's und der Schafe in diesen Gegenden grenzt ans Unglaubliche. Die gesammte Ausfuhr von Häuten betrug im Jahre 1865 mehr als $21 / 2$ Millionen. Rechnet man, dass jährlich 12 Procent des Viehes geschlachtet werden, dann kommt man zu einer Zahl von 22 Millionen Stück, während man aus der Menge ausgeführter Wolle entnimmt, dass in den la Plata Gegenden wenigstens 35 Millionen Schafe vorhanden sein müssen.

Das gesalzene Rindfleisch, unter dem Namen "Charque" bekannt, ist das Hauptnahrungsmittel der Neger, welche sehr daran gewöhnt sind, obwohl es in Beziehung seines Aeussern und des Geschmacks wenig Anlockendes hat. Dieser Ursachen wegen sind auch alle Versuche, dasselbe in Europa einzuführen, fruchtlos gewesen und die in England eingeführten Proben haben selbst das südamerikanische Fleisch daselbst verdächtig gemacht.

Herr Williams, ein „Saladerista “ (Fleischeinsalzer), seit 30 Jahren in Uruguay wohnhaft, ist indess geneigt, das ungünstige Resultat mit dem Fleische in England eher der schlechten Bereitung als der schlechten Beschaffenheit zuzuschreiben. In der Sonne getrocknetes Rindfleisch, wie dies in den la Plata Gegenden bereitet und gut verpackt versendet wird, muss nach seiner Meinung vollkommen gut in Europa ankommen und daselbst eine gute und gesunde Nahrung für die dürftige Klasse ausmachen. Die Bereitungsweise ist sehr einfach. Das Fleisch wird in dünne Streifen geschnitten, in starke Salzlauge getaucht und zwei Tage in Salz gelegt. So bereitet ist es zum Versenden im feuchten 
Zustande geeignet. Wenn man es trocken zu versenden wünscht, muss die Bereitung an offner Luft geschehen und das Fleisch der Sonne ausgesetzt werden, im Sommer 34 Tage, im Winter 4-8 Tage.

Eine Hauptbeschwerde aber gegen diese Sorte von Fleisch ist, dass der grösste Theil seiner nährenden Bestandtheile mit der Salzlauge weggeführt wird und dass durch das Einsalzen die äussersten Fleischtheile beinahe ungeniessbar geworden sind, wenn das Salz erst ins Innerste eingedrungen ist. Dass dies Fleisch übrigens ein gesundes Nahrungsmittel ist, beweisen die kräftige Haltung and die Gesundheit der farbigen Bevölkerung Brasiliens.

Es war also von Wichtigkeit, ein Mittel zu finden, um das Fleisch so zu bereiten, dass es in einem guten essbaren Zustande und zu niedrigen Preisen in Europa eingeführt werden konnte und die Versuche, welche deshalb angestellt wurden, haben zu wichtigen Verbesserungen Anleitung gegeben, welche die erstaunliche Fleisch-Production der südamerikanischen Republiken nicht wenig entwickeln und zugleich eine wahre Wohlthat für die europäische Bevölkerung sein werden.

Die drei Bereitungsmethoden, von denen hier die Rede ist, sind die von John Morgan, Professor in Dublin, v. Liebig in München und Sloper in London.

\section{Morgans Bereitungsmethode.}

Diese Methode hat zu vielen Versuchen geführt, welche aber die ausgezeichnetesten Resultate geliefert haben.

Es ist qu bedauern, dass dieselbe nicht eifriger angewendet wird. Alle Fleischproben, welche nach Liverpool gesendet waren, fanden sogleich Käufer. In einem halben Jahre wurden 500,000 Pfund Rind- und Schaffleisch nach Liverpool gesandt und daselbst gegen 4 Silbergroschen das Pfund verkauft.

Zufolge der Berechnung muss dieser Preis die Kosten der Bereitung kaum decken. Man glaubt aber, dass das Verhältniss sich bessern wird, wenn die Sache einmal geregelt 
in Wirksamkeit ist. Vorzüglich aber, wenn die Unternehmer den Plan von Williams befolgen und Schiffe bauen, welche besonders zu diesem Zwecke eingerichtet und in 4 Abtheilungen getheilt sind. Dadurch würde der Gebrauch von Tonnen, welche grosse Kosten verursachen, überflüssig gemacht.

Das Fleisch, nach Morgan bereitet, ist bis jetzt stets gut und schmackhaft angekommen und ich kann durch persönliche Erfahrung bestätigen, dass die Proben im Aeussern und Geschmack ausgezeichnet waren und dass das Rindfleisch dem englischen (corn-beef) nichts nachgab.

Die Hauptsache bei der Morgan'schen Bereitung ist eine kunstmässige Durchdringung mit Pökel (Salzlauge) mittelst des Circulationssystems im Körper. Sie ist einfach, erfordert wenig Arbeit und keine kostbaren Werkzeuge.

Die Schafe werden durch einen Schlag auf den Kopf getödtet, die Rinder durch einen Messerstich hinter dem Kopfe, welcher den Rückenstrang durchschneidet und unmittelbar den Tod zur Folge hat. Darauf wird die Brust aufgesägt und durch ein Qnerholz aufgehalten, so dass das Herz blosgelegt wird. Alsdann macht man einen Einschnitt in die rechte Herzkammer und einen zweiten in die linke, wodurch das Blut wegströmen kann. Wenn der Blutstrom aufhört, wird eine Rö̀hre mit einem Schliesshahn durch die linke Herzkammer in die grosse Schlagader gebracht, welche das Blut durch den Körper führt und daselbst befestigt.

Diese Röhre wird durch eine biegsame Gutta-Percha Röhre mit einem Gefässe verbunden, worin sich der Pökel befindet. Letzterer besteht aus Wasser und Salz und kleiner Menge Salpeter. Diese Mischung wird in einer Höhe von 18 - 20 Fuss angebracht. Wenn man nun den Pökel abfliessen lässt, durchläuft er das ganze Adersystem, reinigt die Gefässe und Haargefässe und kommt an der rechten Seite des Herzens wieder heraus. Darauf wird die Oeffnung in der rechten Herzkammer geschlossen, wodurch der CirculationsApparat mit allen Gefässen fähig wird, die Einspritzung mit der Conservirungsflüssigkeit zu empfangen. Wenige Secun- 
den reichen hin, um den ganzen Körper auf diese Weise mit der Flüssigkeit zu durchströmen.

Auf diese Weise kann das Fleisch eines Ochsen innerhalb 10 Minuten conservirt werden. Der Druck, womit die Flüssigkeit eingesogen wird, beträgt ungefähr 1 Pfund auf den Quadratzoll und die Menge beträgt 54-63 Quart für jeden Ochsen, für ein Schaf natürlich weniger.

Der Vortheil der Bereitungsweise von Morgan über dem äussern Gebrauche von Salz, besteht vorzüglich darin, dass die natürlichen Säfte und nöhrenden Bestandtheile bewahrt bleiben, während mit der eingespritzten Conservirungs - Flüssigkeit noch andere für die Gesundheit heilsame Bestandtheile in das Fleisch gebracht werden können.

Das so verarbeitete Fleisch wird in Fässer gepackt, deren Jedes ungefähr 110 Pfund enthält, welche in Liverpool 15-20 Thaler kosten.

Das Unternehmen steht unter der Firma: "Morgan Patent Meat-Preserving Company (Limited). Agent der Firma zu Liverpool ist: Irving, 36 Albany, Old Hallstreet.

Diese Firma hat das Privileginm von Herrn Morgan gekauft, und Herr Williams ist in La Plata als Director angestellt. Kürzlich hat die Firma Herm Fleury, Dr. der Medizin aus England, nach La Plata gesendet, um Aufsicht über die Arbeiten des Etablissements zu halten. Dies Etablissement liegt am Ufer des Uruguay, dicht bei der Stadt Paysandu, Republik Uruguay.

Bereitungsweise nach J. von Liebig.

Nicht weit von der Stadt Paysandu, an dem Ufer desselben Stroms bei der kleinen Stadt Fray-Bentos ist die Fabrik zur Bereitung des v. Liebig'schen Extractum carnis.

Die Bereitung unterscheidet sich wesentlich von der Morgan'schen, indem aus dem Fleische ein concentrirtes Extract bereitet wird, welches nur zu Suppen angewendet wird. Wie kräftig dies Extract ist, kann man erwägen, wenn man bedenkt, dass 16 Pfund Fleisch ungefähr $1 / 2$ Pfund 
Extract geben, welches hinreichend ist, um für 128 Personen Bouillon zu liefern. 4 Pfund Extract enthalten die concentrirten nährenden Bestandtheile eines ganzen Ochsen und liefern 1000 Teller kräftige, gute Suppe. Der geringe Umfang dieses Nahrungsmittels macht es ausgezeichnet geschickt für militairischen Grebrauch oder den Seedienst. Es ist ganz frei von Fett, rein und deshalb auch sehr gut für Kranke und zum Gebrauche in Hospitälern.

Bis heute ist dies Extract beinahe ausschliesslich nach Deutschland gesendet worden, wo der Gebrauch bereits sehr ausgebreitet ist. Jetzt ist aber eine neue Unternehmung in London unter dem Namen Liebig s Extract of Meat Company (Limited) 43 Mark-Lane entstanden, welche eine grössere Ausbreitung erhalten wird.

$\mathrm{Zu}$ Fray-Bentos werden täglich durchschnittlich 350 Stück Vieh geschlachtet. Die Bereitungsweise des Extracts ist einfach, erfordert aber viel Arbeit, Sorge und Hiulfe an Werkzeugen.

Das Fleisch muss nach dem Schlachten 24 Stunden abkühlen. Darauf wird es zwischen runden eisernen Rollen (von Innen mit Spitzen versehen) gebracht, welche durch Dampfkraft in Bewegung gesetzt werden, und zu Brei verarbeitet. Dieser Brei wird in ein grosses Gefäss geworfen, worin sich Wasser befindet, während einer Stunde damit verarbeitet und dann in einen grossen Trog, worin sich am Boden ein Sieb befindet, gebracht. Hier läuft der Fleischsaft in ein anderes Gefäss, worin er vom Fett befreit wird. Die reine Fleischflüssigkeit bringt man nun in offene Gefässe, welche mit Dampfröhren versehen sind, so wie mit Blasebälgen, welche einen Luftstrom auf der Oberfläche hervorbringen, um die Feuchtigkeit zu entfernen. Hierdurch wird die Verdampfung befördert. In diesen Gefässen bleibt es 6-8 Stunden, worauf es in einen Filtrir - Apparat gebracht wird. Nach dem Filtriren ist es so dick, dass es in Kruken gefüllt und versendet werden kann. 
Bereitungsmethode nach Sloper.

Diese verdient ebenfalls Berücksichtigung. Die Herren Paris und Sloper zu Buenos Ayres haben die Versuche übernommen und wenn dieselben günstig ausfallen, soll in England eine grosse Fabrik errichtet werden.

Diese Herren versichern, dass sie im Stande sind, Fleisch im frischen Zustande zu conserviren und so nach England zu senden, dass es als frisches Fleisch ankommt und gegen $2 \frac{1}{2}$ bis 3 Sgr. pro Pfund verkauft werden kann. Ueberdies soll dasselbe, wenn es aus den Blechtrommeln genommen ist und der Luft ausgesetzt wird, zweimal so lange frisch bleiben, als das Fleisch der Fleischerläden.

Die Bereitungsweise ist einfach und soll auf der Wegnahme des Sauerstoffs aus der Trommel bestehen, worin das Fleisch verpackt wird. Alle Knochen werden aus dem Fleische entfernt, aber das Fett wird darin gelassen.

Die Luft wird aus den Trommeln durch Wasser gezogen, welches durch den Boden hineingebracht wird und nachdem es den obern Rand erreicht hat, wieder fällt und abläuft. Der leere Raum, welcher dadurch entsteht, wird von oben her durch ein gewisses Gas gefüllt, dessen Zusammensetzung geheim gehalten wird. Dabei muss vorzüglich darauf gesehen werden, dass keine Oeffnung in der Blechtrommel entsteht; denn die kleinste lässt das Gas entweichen und die atmosphärische Luft hineinkommen, wodurch unvermeidlich Verderben entsteht.

Binnen kurzem denken die Unternehmer eine Quantität Fleisch von 5000-6000 Pfund, auf diese Weise bereitet, nach England zu senden. 